\title{
ASOCIACIÓN DEL VALOR GENÉTICO DEL TORO CON CARACTE- RES PRODUCTIVOS EN VACAS LECHERAS EN COLOMBIA
}

\author{
ASSOCIATION OF GENETIC BREEDING VALUE OF THE BULL WITH PRODUCTION \\ TRAITS IN DAIRY COWS IN COLOMBIA
}

\author{
Arango, J. ${ }^{1}$ y Echeverri, J.J. ${ }^{1 *}$ \\ Universidad Nacional de Colombia. Sede Medellín. Grupo BIOGEM. Colombia. *jjecheve@unal.edu.co
}

\author{
Palabras clave adicionales \\ Habilidad de transmisión predicha.
}

\section{RESUMEN}

Actualmente en Colombia no existe un programa que permita la estimación de valores genéticos en bovinos de leche. El objetivo de esta investigación fue determinar la asociación de los EBV (Valor de Cría Estimado) de toros Holstein y Jersey,con el desempeño fenotípico de sus hijas en Colombia; para esto se analizó la asociación de sus EBV con algunos parámetros productivos y sanitarios analizados directamente en su progenie (hijas). Para la estimación de los componentes de varianza, parámetros genéticos y valores genéticos, se utilizó el método de máxima verosimilitud restringida (REML) empleando un algoritmo libre de derivadas con el software MTDFREML. La asociación de las características con los EBV de los toros se realizó utilizando un modelo lineal mixto. Las heredabilidades para producción de leche, proteína, grasa y células somáticas en raza Holstein fueron: $0,19 \pm 0,0 ; 0,37 \pm 0,04 ; 0,37 \pm 0,04 \mathrm{y}$ $0,34 \pm 0,05$ y en Jersey fueron: $0,20 \pm 0,0 ; 0,64 \pm 0,0$; $0,55 \pm 0,0$ y $0,14 \pm 0,26$ respectivamente. Los EBV de los toros Holstein influyeron $(p<0,01)$ sobre la producción, proteína y grasa de la leche. El valor genético también se asoció al conteo de células somáticas. En la raza Jersey se encontraron resultados similares. Los resultados demostraron que a pesar de las bajas confiabilidades estimadas en las evaluaciones en Colombia, las productividades han sido mejoradas con la utilización de genética extranjera.

\section{SUMMARY}

Actually, in Colombia there is not a genetic

\author{
AdDitiOnAL KEYWORDS \\ Predicted transmitting ability.
}

improvement program to estimate breeding values in dairy cattle. The objective of this research was to determine the association of EBV (Estimated Breeding Value) of Holstein and Jersey bulls with the phenotypic performance (milk and health traits) measured directly in their daughters in Colombia. To calculate the variance components, genetic parameters and EBV, the restricted maximum likelihood method (RMLM) using a derivative-free algorithm with the MTDFREML software, was used. The association of milk traits with the bulls EBVs was performed using a mixed model. Heritabilities for milk yield, protein percentage, fat and somatic cell score were $0.19 \pm 0.0,0.37 \pm 0.04$, $0.37 \pm 0.04$ and $0.34 \pm 0.05$, respectively for Holstein animals. For the Jersey were $0.20 \pm 0.0 ; 0.64 \pm 0.0$, $0.55 \pm 0.0$ and $0.14 \pm 0.26$ respectively. The EBV of Holstein bulls were highly significant $(p<0.01)$ on milk production, milk protein and milk fat content. The EBV was also found associated with somatic cell count. Similar results were found in Jersey bulls. The results showed that despite the low reliabilities estimated in Colombia assessments, the productivities are enhanced with the use of foreign genetic.

\section{INTRODUCCIÓN}

Las evaluaciones genéticas son la principal herramienta para lograr que los procesos de selección de animales en los sistemas de producción pecuaria otorguen los resultados esperados en términos de mejoramiento fenotípico de las características de 


\section{ARANGOYECHEVERRI}

interés económico (Echeverri et al., 2008). En la mayoría de las regiones de Colombia, no se cuenta con programas de mejoramiento genético serios y confiables que permitan la selección de sementales con base en el desempeño de sus hijas en el ambiente tropical de este país, esta situación ha ocasionado un flujo de genes de animales de alto mérito, pero seleccionados bajo condiciones ambientales totalmente diferentes, situación que provoca un retraso tecnológico por el desconocimiento de la interacción genotipo-ambiente y por lo que este componente puede generar en el desempeño de la descendencia en ambientes diferentes (Echeverri et al., 2008; Abubakar et al., 1987; Cerón et al., 2001; Echeverri y Quijano, 2001). Las condiciones de realización de las pruebas genéticas de los toros difieren en la mayoría de los hatos colombianos. Por esta razón es de suponer que los resultados obtenidos en los ambientes específicos de cada explotación, no necesariamente coinciden con los resultados de las pruebas en las cuales se basó la selección y compra de los sementales. Un indicativo alto de la efectividad del uso de esta genética foránea, es el análisis del potencial que tiene el reproductor para contribuir a la mejora de ciertas características a través del rendimiento de sus hijas.

El objetivo de esta investigación fue determinar la asociación de los EBV de algunos toros Holstein y Jersey, con el desempeño fenotípico de sus hijas en Colombia.

\section{MATERIAL YMÉTODOS}

La información utilizada procede de 135 hatos bovinos de diferentes regiones del departamento de Antioquia (Colombia). Obteniendo información de la raza Holstein en 2232 lactancias para producción de leche, 1629 para porcentaje de proteína, 1579 para porcentaje de grasa y 1581 para recuento de células somáticas; para la raza Jersey se utilizaron para dichas variables 266,177 , 173 y 176 lactancias, respectivamente. Se emplearon los EBV para 451 toros (389 Holstein y 62 Jersey) para llevar a cabo la asociación.

Los hatos fueron incluidos en un programa de control lechero desde 2008 a 2011 para determinar los parámetros productivos y sanitarios de mayor importancia (Echeverri et al., 2010).

En los hatos con ordeño mecánico la producción de leche fue medida directamente en medidores de paso proporcional True test ${ }^{\circledR}$ y en los hatos con ordeño manual se realizó un pesaje en balde con báscula de precisión en ambos ordeños.

Se tomaron muestras de leche de unos 50 $\mathrm{mL}$ en un recipiente con Bronopol®. Los porcentajes de grasa, proteína y el recuento de células somáticas fueron determinados, mediante la utilización del MilkoScan FT120 ®. El recuento de células somáticas se transformó a puntaje de células somáticas (Somatic Cell Score) (Dabdoub y Shook, 1984).

\section{Estudio ESTADÍSTICO}

Se utilizó el método de máxima verosimilitud restringida (REML) para la estimación de los componentes de varianza y parámetros genéticos, empleando un algoritmo libre de derivadas con el software MTDFREML (Boldman et al., 1995). Para la estimación de los EBV se utilizó un modelo univariado para cada una de las características y la siguiente representación matricial del modelo (Henderson, 1984).

$$
\mathrm{Y}=\mathrm{Xi} \alpha+\mathrm{Xj} \beta+\mathrm{Xk} \varepsilon+\mathrm{XI} \Omega+\mathrm{Xm} \Upsilon+\mathrm{Zm} \varphi+\mathrm{e}
$$

donde:

$Y=$ vector de observaciones para la producción de leche por lactancia;

$\mathrm{X} \mathrm{i}=$ es la matriz de diseño relacionada al efecto fijo del año de parto $\alpha$;

$\mathrm{X} \mathrm{j}=$ es la matriz de diseño relacionada al efecto fijo mes de parto $\beta$;

$\mathrm{Xk}=$ es la matriz de diseño relacionada al efecto fijo de la región $\varepsilon$;

$\mathrm{Xl}=$ es la matriz de diseño relacionada al efecto fijo del grupo contemporáneo (el grupo contem- 


\section{ASOCIACIÓN DE VALORES DE CRÍA CON CARACTERÍSTICAS PRODUCTIVAS}

poráneo fue conformado por los efectos del hato y el numero de parto) $\Omega$;

$\mathrm{Xm}=$ es la matriz de diseño que relaciona el efecto fijo y el coeficiente de regresión desconocidos de la covariable duración de la lactancia $r$;

$\mathrm{Zm}=$ matriz de incidencia que relaciona al efecto aleatorio genético aditivo del animal $\varphi$;

$\mathrm{e}=$ efecto aleatorio residual.

$\mathrm{Y}=\mathrm{Xi} \alpha+\mathrm{Xj} \beta+\mathrm{Xk} \varepsilon+\mathrm{XI} \Omega+\mathrm{Xm} \Upsilon+\mathrm{Xof}+\mathrm{Zm} \varphi+\mathrm{e}$

donde:

$Y=$ vector de observaciones para el promedio de porcentaje de grasa de la leche por lactancia;

$\mathrm{X} \mathrm{i}=$ es la matriz de diseño relacionada al efecto fijo del año de parto $\alpha$;

$\mathrm{Xj}=$ es la matriz de diseño relacionada al efecto fijo mes de parto $\beta$;

$\mathrm{Xk}=$ es la matriz de diseño relacionada al efecto fijo de la región $\varepsilon$;

$\mathrm{XI}=$ es la matriz de diseño relacionada al efecto fijo del grupo contemporáneo $\Omega$;

$\mathrm{Xm}=$ es la matriz de diseño que relaciona el efecto fijo y el coeficiente de regresión desconocidos de la covariable duración de la lactancia $r$;

$\mathrm{Xo}=$ es la matriz de diseño que relaciona el efecto fijo y el coeficiente de regresión desconocidos de la covariable producción real de leche $f$;

$Z m=$ matriz de incidencia que relaciona al efecto aleatorio genético aditivo del animal $\varphi$;

$\mathrm{e}=$ efecto aleatorio residual.

$\mathrm{Y}=\mathrm{Xi} \alpha+\mathrm{Xj} \beta+\mathrm{Xk} \varepsilon+\mathrm{XI} \Omega+\mathrm{Xm} r+\mathrm{Xof}+\mathrm{Zm} \varphi+e$

donde:

$\mathrm{Y}=$ vector de observaciones para el promedio de SCS por lactancia;

$\mathrm{X}=$ es la matriz de diseño relacionada al efecto fijo del año de parto $\alpha$;

$\mathrm{Xj}=$ es la matriz de diseño relacionada al efecto fijo mes de parto $\beta$;

$\mathrm{Xk}=$ es la matriz de diseño relacionada al efecto fijo de la región $\varepsilon$;

$\mathrm{XI}=$ es la matriz de diseño relacionada al efecto fijo del grupo contemporáneo $\Omega$;

$\mathrm{Xm}=$ es la matriz de diseño que relaciona el efecto fijo y el coeficiente de regresión desconocidos de la covariable duración de la lactancia r;
$\mathrm{X}_{0}=$ es la matriz de diseño que relaciona el efecto fijo y el coeficiente de regresión desconocidos de la covariable producción real de leche $f$;

$\mathrm{Zm}=$ matriz de incidencia que relaciona al efecto aleatorio genético aditivo del animal $\varphi$;

$\mathrm{e}=$ efecto aleatorio residual.

En los tres modelos anteriores, debido a la estructura de los datos y al número de niveles para los efectos del hato y el número de parto, estos fueron tratados como grupo contemporáneo, el número de partos fue agrupado en 3 niveles: $1: 1$ y 2 partos; $2: 3,4$ y 5 partos y 3: 6 o más partos. En los modelos definitivos no se incluyó el efecto del ambiente permanente debido a que por la naturaleza de la información y teniendo en cuenta que el programa de control de producción estaba apenas iniciado con el comienzo de este proyecto, muy pocos animales tenían información de varias lactancias, lo cual no permitió incluir este efecto en los modelos.

La región fue incluida como efecto fijo y consistió en un agrupamiento de los municipios en dos niveles de acuerdo con el nivel de especialización en los sistemas de producción lechera de los mismos (1, alta especialización y 2 , media o baja especialización).

$\mathrm{Y}=\mathrm{Xi} \alpha+\mathrm{Xj} \beta+\mathrm{Xk} \varepsilon+\mathrm{XI} \Omega+\mathrm{Xn} \sigma+\mathrm{Xm} \Upsilon+\mathrm{Xof}+$ $Z \mathrm{~m} \varphi+\mathrm{e}$

donde:

$Y=$ vector de observaciones para el promedio del porcentaje de proteína de la leche por lactancia;

$\mathrm{X}=$ es la matriz de diseño relacionada al efecto fijo del hato $\alpha$;

$\mathrm{Xj}=$ es la matriz de diseño relacionada al efecto fijo del número de partos $\beta$;

$\mathrm{Xk}=$ es la matriz de diseño relacionada al efecto fijo del año de parto $\varepsilon$;

$\mathrm{XI}=$ es la matriz de diseño relacionada al efecto fijo del mes de parto $\Omega$;

$\mathrm{X} n=$ matriz de diseño que relaciona el efecto fijo de la región $\sigma$;

$\mathrm{Xm}=$ es la matriz de diseño que relaciona el efecto fijo y el coeficiente de regresión desconoci-

Archivos de zootecnia vol. 63, núm. 242, p. 229. 


\section{ARANGOYECHEVERRI}

dos de la covariable duración de la lactancia $r$;

$\mathrm{Xo}_{\mathrm{o}}=$ es la matriz de diseño que relaciona el efecto fijo y el coeficiente de regresión desconocidos de la covariable producción real de leche $f$;

$\mathrm{Zm}=$ matriz de incidencia que relaciona al efecto aleatorio genético aditivo del animal $\varphi$; $\mathrm{e}=$ efecto aleatorio residual.

El número de partos y la región fueron agrupados como se describió anteriormente. Cada uno de los modelos fue ejecutado en MTDFREML (Boldman et al., 1995), que determina las soluciones para cada efecto fijo y aleatorio mediante el Mixed Model Equations MME, descrito por Henderson (1984) así:

$$
\left|\begin{array}{cr}
X^{\prime} X & X^{\prime} Z \\
Z^{\prime} X & Z^{\prime} Z+A^{-1} a
\end{array}\right|\left|\begin{array}{l}
b \\
\mu
\end{array}\right|=\left|\begin{array}{c}
X^{\prime} Y \\
Z^{\prime} Y
\end{array}\right|
$$

donde:

$\mathrm{X}=$ es la matriz de diseño que relaciona el efecto fijo;

$Z=$ es la matriz de diseño que relaciona el efecto aleatorio del animal;

$A=$ es la matriz de parentesco;

$b=$ es el vector de soluciones para los efectos fijos y covariables;

$\mathrm{u}=$ es el vector de soluciones para los efectos aleatorios.

$\alpha=\sigma^{2} e / \sigma^{2} a \circ\left(1-h^{2}\right) / h^{2}$

Mediante la estimación de la asociación de los valores genéticos con los caracteres productivos, se prentende lograr el acercamiento al objetivo principal del trabajo que es determinar la efectividad de la utilización de toros importados en Colombia.

El recuento de células somáticas (RCS) determinado en cada control fue transformado a una escala lineal para obtener una puntuación de células somáticas (SCS) de 1 a 9, mediante una transformación logarítmica, según la expresión $\log _{2}(\mathrm{RCS} / 100)+3$, (Dabdoub y Shook ,1984). Las lactancias fueron ajustadas a una duración de 305 días.
Los porcentajes de proteína y grasa se calcularon con base en las lactancias completas que tenían información para las características.

Se determinaron las asociaciones de cada una de las características con los valores de cría de los toros utilizando un modelo mixto, basado en las fuentes de variación conocidas para cada una de las variables dependientes (producción de leche ajustada a 305 días, porcentaje de grasa y proteína y SCS).

El modelo estadístico utilizado fue:

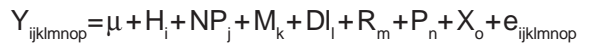

donde:

$\mathrm{Y}_{\mathrm{ijklmnop}}=$ característica dependiente (producción de leche por lactancia, porcentaje de proteína de la leche, porcentaje de grasa de la leche y puntaje de células somáticas);

$\mu=$ media para cada una de las características; $\mathrm{H}_{\mathrm{i}}=$ efecto fijo del hato $\mathrm{i}$;

$\mathrm{NP}_{\mathrm{j}}=$ efecto fijo del numero de parto $\mathrm{j}$;

$\mathrm{M}_{\mathrm{k}}=$ efecto del municipio $\mathrm{k}$;

$\mathrm{DL}_{\mathrm{l}}=$ efecto de la covariable duración de la lactancia I;

$R_{m}=$ efecto de la covariable valor de cría del reproductor $\mathrm{m}$;

$P_{n}=$ efecto de la covariable precisión del valor de cría n;

$\mathrm{X}_{0}=$ efecto de la covariable numero de hijas 0 ; $\mathrm{e}_{\mathrm{ijk} \mid \mathrm{l} n o \mathrm{p}}=$ error experimental.

Para determinar el grado de asociación, se estimaron las correlaciones entre los valores de cría de los toros con cada una de las características, por medio de la correlación de rangos de Spearman, con la ayuda de paquete estadístico SAS 9.2 (SAS Institute, 2009); también se realizó un análisis de regresión de los valores genéticos con el valor fenotípico de las hijas para cada una de las variables. El modelo general utilizado para este análisis fue el siguiente:

$$
Y=\beta_{0}+\beta_{1} X_{i}
$$

donde:

$\mathrm{Y}=$ variable dependiente (producción de leche 


\section{ASOCIACIÓN DE VALORES DE CRÍA CON CARACTERÍSTICAS PRODUCTIVAS}

ajustada, porcentaje de grasa, porcentaje de proteína o puntaje de células somáticas);

$\beta_{0}=$ intercepto;

$\beta_{1}=$ es el coeficiente de regresión estimado para el EBV del toro para cada característica; $\mathrm{X}_{\mathrm{i}}=$ valor de cría del toro.

\section{RESULTADOSYDISCUSIÓN}

\section{ANÁLISIS DESCRIPTIVO}

La descripción estadística de los registros de producción de leche ajustada a 305 días, porcentaje de proteína, grasa y puntaje de células somáticas para la raza Holstein y Jersey se presentan en la tabla I. Los animales Holstein presentaron una media para producción de leche de $5471 \pm 1787 \mathrm{~kg}$ / lactación, mayor que los animales Jersey, quienes presentaron una media de $4164 \pm$ 2051 kg/lactación, estos resultados concuerdan con lo reportado por otros autores (Mcallister, 2002; Bolívar et al., 2009), ya que entre los factores genéticos, la raza es uno de los más importantes en la variación de producción de leche entre individuos. En cuanto a la composición de la leche, la raza Jersey mostró superioridad en el porcentaje de proteína $(3,46 \pm 0,33)$ y grasa $(4,69 \pm 0,71)$ con respecto a la Holstein, cuyas medias fueron $3,04 \pm 0,28$ y $3,80 \pm 0,46$, respectivamente. Estos resultados concuerdan con reportes de otros autores (Schutz et al., 1990; Bolívar et al., 2009, Bailey et al., 2005, Teodoro y Madalena, 2005). El coeficiente de variación estimado para la producción de leche y el puntaje de células somáticas en ambas razas fue mayor comparado con los resultados de las demás características, la característica de menor variación en ambas razas fue el porcentaje de proteína de la leche. El puntaje de células somáticas $(4,45 \pm$ $1,34)$ fue mayor en los Holstein que en los Jersey $(4,20 \pm 1,38)$. Mcallister (2002) indica que la raza Jersey tiene más susceptibilidad a la mastitis que la Holstein, lo que no concuerda con los resultados de esta investigación.

\section{ESTIMACIÓN DE PARÁMETROS GENÉTICOS}

En la tabla II se presenta la heredabilidad y los componentes de varianza aditiva, residual y fenotípica para cada una de las características en las razas Holstein y Jersey. Los valores de heredabilidad para producción de leche en las dos razas son similares, pero no para el porcentaje de grasa y proteínas (tabla II) que son mayores en la raza Jersey. Los valores genéticos estimados para producción de leche en ambas razas son notoriamente inferiores a los reportados para toros Americanos y Canadienses en las evaluaciones de sus países de origen. Los valores genéticos estimados para las demás características son más consistentes con las estimaciones extranjeras. Es importante resaltar que las precisiones logradas en esta investigación no son muy altas, debido al bajo numero de hijas por toro de las que se tiene registro en Colombia. Esta situación dificulta el éxito de los programas de mejo-

Tabla I. Estadística descriptiva de características productivas de vacas Holstein y Jersey. (Descriptive statistics for productive characteristics in Holstein and Jersey cows).

\begin{tabular}{lcccccccc}
\hline & \multicolumn{4}{c}{ Holstein } & \multicolumn{4}{c}{ Jersey } \\
& PL & \% proteína & \% grasa & SCS & PL & \% proteína & \% grasa & SCS \\
\hline $\mathrm{n}$ & 2232 & 1629 & 1579 & 1581 & 266 & 177 & 173 & 176 \\
Media & 5351 & 3,04 & 3,80 & 4,45 & 4164 & 3,46 & 4,69 & 4,20 \\
DE & 1787 & 0,28 & 0,46 & 1,34 & 2051 & 0,33 & 0,71 & 1,38 \\
CV & 33,39 & 9,28 & 12,16 & 30,7 & 49,25 & 9,54 & 15,14 & 33,0 \\
\hline
\end{tabular}

$\mathrm{PL}=$ producción de leche, $\mathrm{kg} / \mathrm{lactación} ; \mathrm{SCS}=$ puntaje de células somáticas. 


\section{ARANGOYECHEVERRI}

Tabla II. Heredabilidad y componentes de varianza de características productivas de vacas Holstein y Jersey. (Heritability and variance components for productive characteristics in Holstein and Jersey cows).

\begin{tabular}{lcccccccc}
\hline & \multicolumn{3}{c}{ Holstein } & \multicolumn{3}{c}{ Jersey } \\
& $\mathrm{PL}$ & \% proteína & \% grasa & SCS & $\mathrm{PL}$ & $\%$ proteína & \% grasa & SCS \\
\hline$\sigma^{2} \mathrm{a}$ & 415572 & 0,02 & 0,08 & 460 & 246649 & 0,05 & 0,25 & 51 \\
$\sigma^{2} \mathrm{e}$ & 1782630 & 0,04 & 0,15 & 902 & 985598 & 0,03 & 0,21 & 322 \\
$\sigma^{2} \mathrm{p}$ & 2198202 & 0,06 & 0,23 & 1363 & 1233247 & 0,08 & 0,46 & 373 \\
$\mathrm{~h}^{2}$ & 0,19 & 0,37 & 0,37 & 0,34 & 0,20 & 0,64 & 0,55 & 0,14 \\
$\mathrm{EE}$ & 0,0 & 0,04 & 0,04 & 0,05 & 0,0 & 0,0 & 0,0 & 0,26 \\
\hline
\end{tabular}

$\mathrm{PL}=$ producción de leche, $\mathrm{kg} /$ lactación; $\mathrm{SCS}=$ puntaje de células somáticas.

$\sigma^{2} \mathrm{a}=$ varianza genética aditiva; $\sigma^{2} \mathrm{e}=$ varianza residual; $\sigma^{2} \mathrm{p}=$ varianza fenotípica; $\mathrm{h}^{2}=$ heredabilidad; $\mathrm{EE}=$ error estándar.

ramiento genético en el país. Los resultados mencionados anteriormente, para los 5 mejores toros, se pueden ver en las tablas III y IV. La figura 1 muestra la tendencia en los valores genéticos estimados para las diferentes características presentadas como la frecuencia de los rangos de valores genéticos estimados para las mismas. Estas frecuencias confirman la inferioridad de los valores genéticos estimados ya que muestran una alta frecuencia de los rangos más bajos para producción de leche.

La heredabilidad obtenida para producción de leche para las razas Holstein y Jersey, fue inferior a la reportada por Ojango y Pollott (2001) y Yazgan et al. (2010). En el caso del porcentaje de grasa y proteína las heredabilidades encontradas en esta investigación para la raza Holstein son muy inferiores a las reportadas por otros autores, pero similares a las encontradas en la raza Jersey (Juga, 1992; Hashemi et al., 2009).

Tabla III. Valores de cría de los mejores toros que se obtuvieron para producción de leche, y puntaje de células somáticas. (HPTL) EBV para leche, (HPTSCS) EBV para puntaje de células somaticas y (PRECL) precisión de los valores de cría estimados. (Breeding values of the best bulls that were obtained for milk yield and somatic cell score. (HPTL) EBV for milk, (HPTSCS) EBV for somatic cell score and (PRECL) accuracy of estimated breeding values).

\begin{tabular}{lcccccccccccc}
\hline & \multicolumn{1}{c}{ Holstein } & \multicolumn{1}{c}{ Jersey } \\
Código & HPTL & PRECL & Código HTPSCS PRECL & Código & HPTL & PRECL & Código HPTSCSPRECL \\
\hline C1 & 524 & 0,54 & C6 & $-13,37$ & 0,49 & C11 & 222 & 0,32 & C16 & $-1,26$ & 0,31 \\
C2 & 493 & 0,58 & C7 & $-10,45$ & 0,73 & C12 & 179 & 0,41 & C17 & $-0,80$ & 0,11 \\
C3 & 349 & 0,23 & C8 & $-8,27$ & 0,72 & C13 & 123 & 0,38 & C18 & $-0,73$ & 0,27 \\
C4 & 287 & 0,45 & C9 & $-7,00$ & 0,26 & C14 & 112 & 0,48 & C19 & $-0,73$ & 0,14 \\
C5 & 263 & 0,47 & C10 & $-5,88$ & 0,79 & C15 & 85 & 0,21 & C20 & $-0,66$ & 0,40 \\
\hline
\end{tabular}

$\mathrm{C} 1=007 \mathrm{HO} 05708 ; \mathrm{C} 2=072 \mathrm{HO} 00753 ; \mathrm{C} 3=073 \mathrm{HO} 02479 ; \mathrm{C} 4=029 \mathrm{HO} 08246 ; \mathrm{C} 5=073 \mathrm{HO} 01965 ; \mathrm{C} 6=$ $009 \mathrm{HO} 01895 ; \mathrm{C} 7=\mathrm{NAL} 012 \mathrm{HO} ; \mathrm{C} 8=072 \mathrm{HO} 00753 ; \mathrm{C} 9=\mathrm{NAL} 068 \mathrm{HO} ; \mathrm{C} 10=220 \mathrm{HO} 09600 ; \mathrm{C} 11=001 \mathrm{JE} 01325$; C12=014JE00365; C13= NAL0525ACCGJ; C14=014JE00285; C15=007JE00535; C16=014JE00326; C17=014JE00205; C18=007JE00622; C19=007JE00535; C20=071JE00162.

Archivos de zootecnia vol. 63, núm. 242, p. 232. 
Tabla IV. Valores de cría de los mejores toros Holstein y Jersey que se obtuvieron para porcentaje de proteína y porcentaje de grasa. (HPTPR) EBV para porcentaje de proteína, (HPTG) EBV para porcentaje de grasa y (PRECL) precisión de los valores de cría estimados. (Breeding values of Holstein and Jersey bulls best obtained for protein percentage and fat percentage. HPTPR) EBV for protein percentage (HPTG) EBV for fat percentage and (PRECL) accuracy of estimated breeding values).

\begin{tabular}{ccccccccccccc}
\hline \multicolumn{1}{c}{ Holstein } & \multicolumn{1}{c}{ Jersey } \\
Código & HPTPR & PRECL Código & HTPG & PRECL & Código & HPTPR & PRECL Código & HPTG & PRECL \\
& & & & & & & & & & & \\
\hline C1 & 0,109 & 0,36 & C6 & 0,180 & 0,66 & C11 & 0,103 & 0,48 & C16 & 0,17 & 0,47 \\
C2 & 0,093 & 0,75 & C7 & 0,160 & 0,72 & C12 & 0,100 & 0,38 & C17 & 0,54 & 0,54 \\
C3 & 0,087 & 0,79 & C8 & 0,148 & 0,36 & C13 & 0,086 & 0,27 & C18 & 0,15 & 0,52 \\
C4 & 0,087 & 0,52 & C9 & 0,143 & 0,30 & C14 & 0,079 & 0,58 & C19 & 0,14 & 0,24 \\
C5 & 0,087 & 0,44 & C10 & 0,142 & 0,61 & C15 & 0,064 & 0,50 & C20 & 0,10 & 0,36
\end{tabular}

$\mathrm{C} 1=007 \mathrm{HO} 006120 ; \mathrm{C} 2=007 \mathrm{HO} 06250 ; \mathrm{C} 3=073 \mathrm{HO} 02239 ; \mathrm{C} 4=001 \mathrm{HO} 05306 ; \mathrm{C} 5=\mathrm{PANTA023HO}$; 6 = 202HO00013; C7= 071HO01181; C8= NAL121HO; C9=200HO05127; C10=072HO01758; C11= NZLM000000300507; C12=001JE00321; C13= 200JE00109; C14= DNKM000000302430; C15= 001JE00457; C16=001JE00317; C17 = DNKM000000302430; C18=NAL0525ACCGJ; C19=200JE00109; C20 $=$ PANTA034JE.

Las heredabilidades estimadas para el recuento de células somáticas en ambas razas, es muy superior a las reportadas por otros autores (Yazgan, 2010).

\section{EFECTO DEL EBV DEL TORO SOBRE EL DESEMPEÑO PRODUCTIVO DE LAS HIJAS JERSEY}

El EBV del toro, tuvo efecto $(\mathrm{p}<0,01)$ sobre la producción de leche de sus hijas solo en la primera lactancia. Para porcentaje de proteína el EBV del toro tuvo efecto $(\mathrm{p}<0,01)$ en todas las lactancias. Para el caso del puntaje de células somáticas el EBV del toro presentó efecto $(\mathrm{p}<0,05)$ solo en primera lactancia. El porcentaje de grasa no fue afectado por el EBV del toro. La utilización de estos toros probados asegura que el desempeño de las hijas estará de acuerdo a los EBV reportados por cada uno de los sementales y que el nivel genético por generación logrado mediante un proceso de selección será mayor que el obtenido a través del uso de toros de los que se desconoce el desempeño de su progenie (Echeverri etal., 2008).
Aunque no se realizaron las estimaciones de progreso genético, dado que la confiabilidad de los estimados de EBV hacen parte del acercamiento matemático para la estimación de este, se puede pensar que la alta confiabilidad de los EBV permitiría esperar un progreso genético alto para las características estudiadas. Este estudio es una de las primeras aproximaciones en el país por lo cual es necesario realizar un análisis con un mayor número de datos que confirme estos resultados.

Los coeficientes de regresión de los EBV de los toros de raza Jersey para producción de leche, porcentaje de proteína y células somáticas en cada una de las lactancias evaluadas se muestran en la tabla V. Los coeficientes de regresión para todas las características en la primera lactancia fueron significativos $(\mathrm{p}<0,05)$ indicando que por cada unidad de aumento en el EBV del toro Jersey, la producción de leche aumenta en 5,7 litros, el porcentaje de proteína en $2,1 \%$, el porcentaje de grasa $2,6 \%$ y el puntaje de células somáticas en 0,76. En la segunda lactancia solo el coeficiente de 


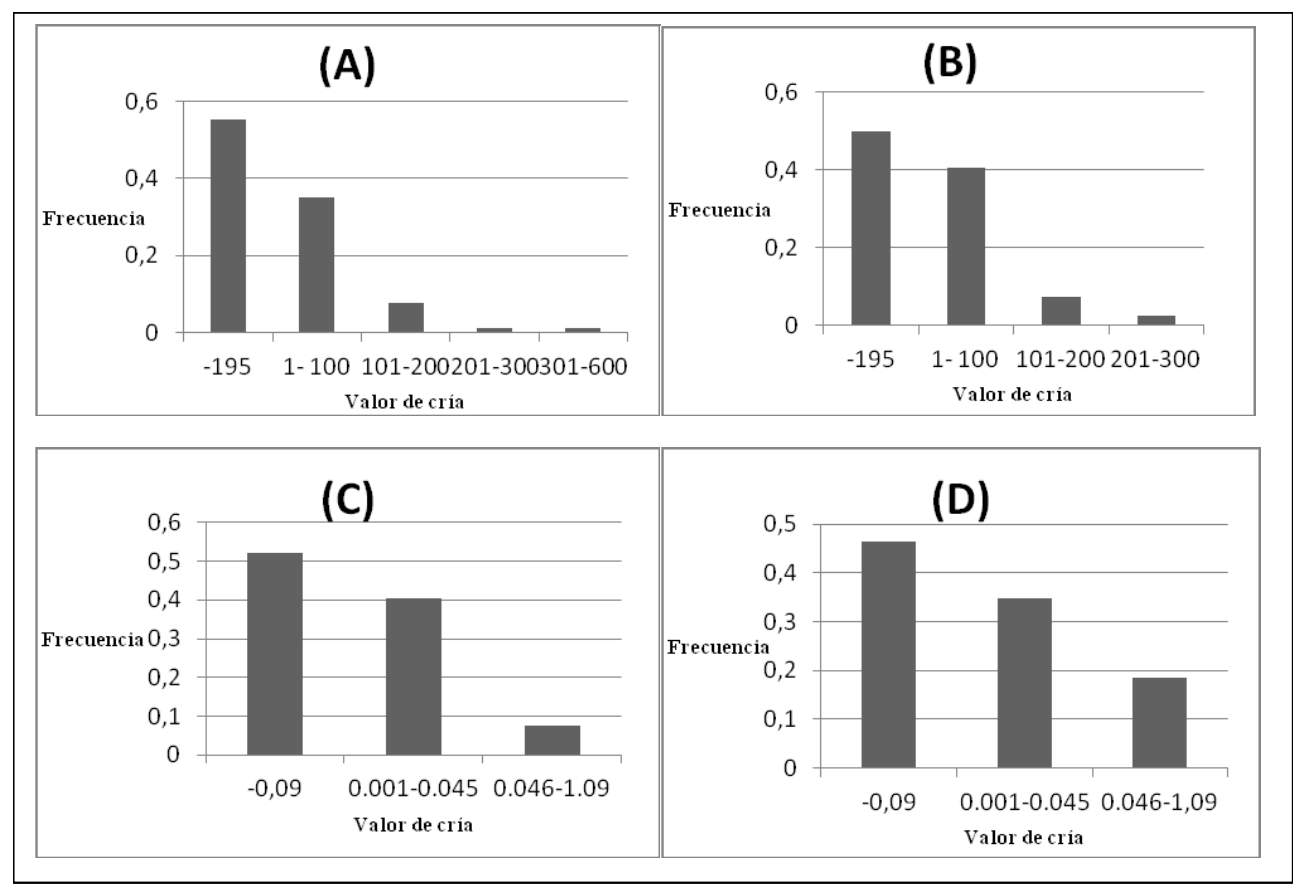

Figura 1. Frecuencias de valores de cría para producción de leche y porcentaje de proteína en Holstein y jersey. (A) EBV para producción de leche en la raza Holstein, (B) EBV para producción de leche raza Jersey, (C) EBV para porcentaje de proteina raza Holstein, (D) EBV para porcentaje de proteína raza Jersey. (Frequency of breeding values for milk yield and protein percentage in Holstein and Jersey. (A) EBV for milk yield in Holsteins, (B) EBV for milk yield Jersey breed, (C) EBV for protein percentage Holstein, (D) EBV for protein percentage Jersey breed).

regresión para porcentaje de proteína fue significativo $(\mathrm{p}<0,05)$, es decir por cada unidad de aumento en EBV el porcentaje de proteína aumenta en un 4,05\%. El coeficiente de regresión del valor de cría para el porcentaje de grasa fue significativo. En la tercera lactancia se detectó que si el EBV aumenta en una unidad, el porcentaje de grasa y la producción de leche suben en un $4,55 \%$ y 8,87 litros respectivamente. Resultados que indican que el efecto mejorador de estos reproductores sobre dichas características excepto para el puntaje de células somáticas que se espera disminuya en el desempeño de las hijas.

La correlación de los EBV de los toros Jersey con la producción de leche de las hijas fue de 0,39 ( $p<0,01)$. Esta asociación fue mayor cuando se estimó en la primera lactancia. La correlación para el porcentaje de proteína con los EBV de los toros fue de $0,27$ ( $p>0,05)$, esta asociación fue mayor cuando se evaluó en la segunda lactancia $0,46$ ( $p<0,01)$. La correlación para el porcentaje de grasa y puntaje de células no fueron significativas (tabla VI).

\section{EFECTO DEL EBV DEL TORO SOBRE EL DESEMPEÑO PRODUCTIVO DE LAS HIJAS HOLSTEIN \\ Salvo en la tercera lactancia, el EBV del toro afectó $(\mathrm{p}<0,01)$ a la producción de leche de sus hijas. Asimismo, el EBV del toro tuvo efecto $(\mathrm{p}<0,05)$ sobre la producción de pro-}




\section{ASOCIACIÓN DE VALORES DE CRÍA CON CARACTERÍSTICAS PRODUCTIVAS}

Tabla V. Coeficientes de regresión entre los valores de cría de los toros Jersey y el desempeño productivo y sanitario de sus hijas: leche (HPTL); porcentaje de proteina (HPTPR); porcentaje de grasa (HPTG) y puntaje de células somáticas (HPTRCS). (Regression analysis of the breeding values of Jersey bulls in each lactation of their daughters: milk (HPTL); protein percentage (HPTPR); fat percentage (HPTG) and somatic cell score (HPTRCS)).

\begin{tabular}{lcccc}
\hline No & HPTPL & HPTPR & HPTG & HPTRCS \\
\hline L1 & 5,71 & 2,71 & 2,86 & 0,76 \\
$\mathrm{p}$ & 0,0006 & 0,0056 & 0,008 & 0,0132 \\
$\mathrm{~L} 2$ & 3,41 & 4,05 & 1,58 & 0,33 \\
$\mathrm{p}$ & 0,14 & $<0,0001$ & 0,29 & 0,175 \\
$\mathrm{~L} 3$ & 8,87 & 2,48 & 4,55 & 0,247 \\
$\mathrm{p}$ & 0,0030 & 0,056 & 0,005 & 0,465 \\
L6 o más & 5,63 & 4,8 & 1,36 & $-1,21$ \\
$\mathrm{p}$ & 0,48 & 0,04 & 0,69 & 0,20 \\
\hline
\end{tabular}

$\mathrm{L}=$ lactación

teína, menos en la cuarta lactancia. El EBV del toro para porcentaje de grasa tuvo efecto $(\mathrm{p}<0,01)$ en todas las lactancias. Para el caso de células somáticas el valor de cría del toro tuvo efecto $(\mathrm{p}<0,05)$ solo hasta la tercera lactancia.

Los coeficientes de regresión entre los EBV de los toros Holstein y los valores fenotípicos de sus hijas, se presentan en la tabla VII. ElEBV del toro afectó a la producción de leche en la primera y segunda lactancia, indicando que por cada unidad de aumento del valor de cría la producción de leche aumenta en 1,36 y 2,21 litros; lo que concuerda con resultados encontrados por otros autores (Stanton et al., 1991) quienes reportan que la respuesta en el desempeño de las hijas es mayor en vacas primíparas en Colombia y en las vacas de menos de dos partos en México.

Para porcentaje de proteína y grasa los coeficientes de regresión fueron significativos en todas las lactancias y para el re- cuento de células somáticas solo en la segunda lactancia, resultando en un aumento de 6 en el puntaje del recuento por una unidad de aumento en el EBV, resultados que concuerdan con los de Schutz et al. (1990) que encontraron que el puntaje de células somáticas aumenta con el número de partos del animal.

Las correlaciones entre los valores de cría de toros Holstein y fenotípicos de las hijas, se muestran en la tabla VII. La correlación fue significativa para la producción de leche en las dos primeras lactancias, encontrándose mayor grado de asociación en el primer parto $19 \%$, para el porcentaje de proteína se encontró asociación con el EBV del toro en un $28 \%$ para la primera lactancia, en la segunda y tercera lactancia $34 \%$, y en la sexta o en lactancias superiores a ésta, en un $30 \%$ (tabla VIII). El valor de cría y el valor fenotípico para el porcentaje de grasa fueron significativos en todas las lactancias encontrándose mayor valor de asociación en la tercera. Para el puntaje de células somáticas

Tabla VI. Correlaciones de los valores cría de toros de raza Jersey con los valores fenotípicos de sus hijas: leche (HPTL); porcentaje de proteína (HPTPR); porcentaje de grasa (HPTG) y puntaje de células somáticas (HPTRCS). (Correlations of breeding values of Jersey bulls with the phenotypic values of their daughters: milk (HPTL); protein percentage (HPTPR); fat percentage (HPTG) and somatic cell score (HPTRCS)).

\begin{tabular}{lcccc}
\hline № & HPTPL & HPTPR & HPTG & HPTRCS \\
\hline L1 & 0,39 & 0,27 & 0,24 & 0,29 \\
p & 0,0003 & 0,057 & 0,08 & 0,053 \\
L2 & 0,23 & 0,46 & 0,0039 & 0,15 \\
p & 0,04 & 0,0005 & 0,97 & 0,29 \\
L3 & 0,20 & 0,36 & 0,55 & 0,14 \\
p & 0,16 & 0,04 & 0,001 & 0,49 \\
L6 o más & 0,21 & 0,17 & 0,31 & $-0,767$ \\
p & 0,45 & 0,63 & 0,372 & 0,01 \\
\hline
\end{tabular}

$\mathrm{L}=$ lactación 


\section{ARANGOYECHEVERRI}

Tabla VII. Coeficientes de regresión de los EBV de los toros Holstein y el desempeño productivo y sanitario de sus hijas: leche (HPTL); porcentaje de proteína (HPTPR); porcentaje de grasa (HPTG) y puntaje de células somáticas (HPTRCS). (Regression analysis of the EBV of Holstein bulls in each lactation of their daughters: milk (HPTL); protein percentage (HPTPR); fat percentage (HPTG) and somatic cell score (HPTRCS)).

\begin{tabular}{lcccc}
\hline No & HPTPL & HPTPR & HPTG & HPTRCS \\
\hline L1 & 1,36 & 1,78 & 1,12 & 0,04 \\
p & 0,05 & $<0,0001$ & 0,0072 & 0,122 \\
L2 & 2,21 & 2,32 & 2,28 & 0,066 \\
p & 0,0005 & $<0,0001$ & $<0,0001$ & 0,0011 \\
L3 & 0,26 & 1,90 & 2,22 & 0,020 \\
p & 0,69 & $<0,0001$ & $<0,0001$ & 0,28 \\
L6 o más & 2,09 & 1,99 & 1,92 & 0,05 \\
$p$ & 0,14 & $<0,0001$ & $<0,0001$ & 0,14 \\
\hline
\end{tabular}

$\mathrm{L}=$ lactación

elEBV no se correlacionó significativamente.

Algunos estudios sobre la respuesta de selección de toros de EEUU en América latina (Stanton et al., 1991) muestran que la producción de leche de vacas Holstein es generalmente menor en América Latina que en los EEUU, ya que la habilidad de transmisión predicha de los sementales seleccionados no tiene por qué ser la misma en ambientes distintos (Stanton et al., 1991; Falconer y Mackay, 1996; Kolmodin, 2003).

Sin embargo, las estimaciones de la primera, segunda y tercera lactancias fueron las más acertadas; incluso en algunos de los resultados se obtiene una asociación mayor de los EBV cuando se estiman en la primera y segunda lactancia.

\section{CONCLUSIONES}

La utilización de genética foránea ha tenido un fuerte impacto en la productividad de los hatos lecheros en Colombia; a pesar de haber utilizado toros evaluados genéticamente en ambientes diferentes, se ha logrado obtener mejoras productivas a lo largo del tiempo.

Los individuos de mejor desempeño fenotípico en Colombia, corresponden a las vacas hijas de toros con valores genéticos superiores calculados en otras latitudes.

Los toros con mayores valores genéticos han dado lugar a hijas más productivas para las características evaluadas.

Las características que han presentado mejor respuesta con respecto a la selección de toros foráneos, han sido las productivas y de composición de la leche, la respuesta de las características sanitarias fue un poco menos consistente.

Para las dos razas de mayor importancia en Colombia los resultados fueron similares, indicando mejoras productivas en las hijas de toros con valores genéticos mejores para las características productivas y composicionales y resultados menos consistentes en el tema de caracteres sanitarios.

Tabla VIII. Correlaciones de los valores cría de toros de raza Holstein con los valores fenotípicos de sus hijas: leche (HPTL); porcentaje de proteína (HPTPR); porcentaje de grasa (HPTG) y puntaje de células somáticas (HPTRCS). (Correlations of breeding values of Holstein bulls with the phenotypic values of their daughters: milk (HPTL); protein percentage (HPTPR); fat percentage (HPTG) and somatic cell score (HPTRCS)).

\begin{tabular}{lcccc}
\hline No & HPTPL & HPTPR & HPTG & HPTRCS \\
\hline L1 & 0,19 & 0,28 & 0,18 & 0,040 \\
p & $<, 0001$ & $<, 0001$ & 0,0004 & 0,424 \\
L2 & 0,12 & 0,34 & 0,29 & 0,084 \\
p & $<, 0001$ & $<, 0001$ & $<, 0001$ & 0,0759 \\
L3 & $-0,038$ & 0,34 & 0,32 & 0,047 \\
p & 0,42 & $<, 0001$ & $<, 0001$ & 0,3994 \\
L6 o más & 0,18 & 0,30 & 0,31 & 0,20 \\
P & 0,032 & 0,0018 & 0,0013 & 0,047 \\
\hline
\end{tabular}

$\mathrm{L}=$ lactación 


\section{ASOCIACIÓN DE VALORES DE CRÍA CON CARACTERÍSTICAS PRODUCTIVAS}

\section{BIBLIOGRAFÍA}

Abubakar, B.; Mcdowell, R. y Van, L. 1987. Evaluación genética de los Holstein en Colombia. Rev Holstein, 98: 37-40.

Bailey, K.; Jones, C. and Heinrichs, A.J. 2005 Economic returns to Holstein and Jersey herds under multiple component pricing. J Dairy Sci, 88: 2269-2280.

Boldman, K.G.; Kriese, L.A.; Van Vleck, L.D.; Van Tassel, C.P. and Kachman, S.D. 1995. A Manual for Use of MTDFREML. A set of programs to obtain estimates of variances and covariances. U.S. Department of Agriculture. Agricultural Research Service.

Bolívar, D.; Echeverri, J.; Restrepo, L. y Cerón, M. 2009. Productividad de vacas Jersey, Holstein y Jersey*Holstein en una zona de bosque húmedo montano bajo (Bh-MB). Livest Res Rural Dev, 21: 80.

Cerón, M.; Tonhati, H.; Costa, C. y Benavides, O. 2001. Interacción genotipo-ambiente en ganado Holstein colombiano. Arch Latinoam Prod Anim, 9: 74-78.

Dabdoub, S.M. and Shook, G.E. 1984. Phenotypic relations among milk yield, somatic cell count and clinical mastitis. J Dairy Sci, 67: 163-164.

Echeverri, J. y Quijano, J. 2001. Estimación de algunos parámetros genéticos y ambientales para el porcentaje de proteína en la leche. Despertar lechero, 19: 125-135.

Echeverri, J.; Parra, J.; López, A. y Quijano, J. 2008. Programas de control lechero evaluaciones genéticas y su importancia en Colombia. Memorias VI Seminario Internacional Competitividad en Carne y Leche. Colombia. pp. 183187.

Echeverri, J.; López, A. y Parra, J. 2010. Software control 1 Manejo y control de producción para hatos lecheros. Ed. Unal. Colombia.

Falconer, D. and Mackay, F. 1996. Introduction to quantitative genetics. $4^{\text {th }}$ ed. Longman Green.
Harlow, Essex. UK.

Hashemi, A.;Bernousi, I.;Zadeh, S.R. and Ranjbari, M. 2009. Estimates of genetic parameters of productive traits in Holstein-native crossbreeds in W. Azerbaijan province-Iran. J Anim Vet Adv, 8: 917-919.

Henderson, C. 1984. Applications of linear models in animal breeding. University of Guelph. Ontario. pp. 423.

Juga, J. 1992. Estimation of variances and covariances of milk traits by REML with an in-dividual animal model. Acta Agr Scand, 42: 198-204.

Kolmodin, R. 2003. Genotype by enviroment interaction in nordic dairy cattle studied using reaction norms. Acta Agr Scand A-AN, 52: 1124.

Mcallister, A.J. 2002. Is crossbreeding the answer to questions of dairy breed utilization?. J Dairy Sci, 85: 2352-2357.

Ojango, J. and Pollott, G. 2001. Genetics of milk yield and fertility traits in Holstein-Friesian cattle on large-scale. J Anim Sci, 79: 1742-1750.

SAS Institute. 2009. 9.2 SQL Procedure User's Guide. SAS Institute. Inc. Cary, N.C. USA.

Schutz, M.; Hanse, L. and Steuernagel, G. 1990. Variation of milk, fat, protein, and somatic cells for dairy cattle. J Dairy Sci, 73: 484-493.

Stanton, T.; Blake, R. and Quaas, R.L. 1991 Response to selection of United States Holstein sires in Latin America. J Dairy Sci, 74: 651- 664.

Teodoro, R.L. and Madalena, F.E. 2005. Evaluation of crosses of Holstein, Jersey or Brown Swiss sires x Holstein-Friesian/Gir dams. 3. Lifetime performance and economic evaluation. Genet Mol Res, 4: 84-93.

Yazgan, K.; Makulska, J.; Weglarz, A.; Ptak, E. and Gierdziewicz, M. 2010. Genetic relationship between milk dry matter and other milk traits in extended lactations of Polish Holstein cows. Czech J Anim Sci, 55: 91-104. 